\title{
The role of nutraceuticals and phytotherapy in the management of urinary tract infections: What we need to know?
}

\author{
Tommaso Cai ${ }^{1}$, Irene Tamanini ${ }^{1}$, Ekaterina Kulchavenya ${ }^{2}$, Tamara Perepanova ${ }^{3}$, Béla Köves ${ }^{4}$, \\ Florian M.E. Wagenlehner ${ }^{5}$, Zafer Tandogdu ${ }^{6}$, Gernot Bonkat ${ }^{7}$, Riccardo Bartoletti ${ }^{8}$, \\ Truls E. Bjerklund Johansen ${ }^{9}$ \\ ${ }^{1}$ Department of Urology, Santa Chiara Regional Hospital, Trento, Italy; \\ ${ }^{2}$ TB Research Institute, Medical University, Novosibirsk, Russian Federation; \\ ${ }^{3}$ N. Lopatkin Scientific Research Institute of Urology and Interventional Radiology - branch of the National Medical Research \\ Radiological Centre of the Ministry of Health of the Russian Federation; \\ ${ }^{4}$ Jahn Ferenc South Pest Teaching Hospital, Budapest, Hungary; \\ ${ }^{5}$ Klinik und Poliklinik für Urologie, Kinderurologie und Andrologie, Universitätsklinikum Giessen und Marburg GmbH, \\ Justus-Liebig-Universität, Giessen, Germany; \\ ${ }^{6}$ Northern Institute for Cancer Research, Newcastle University, NE1 7RU Newcastle upon-Tyne, UK; \\ ${ }^{7}$ Alta uro AG, Merian Iselin Klinik, Center of Biomechanics \& Calorimetry (COB), University Basel, Basel, Switzerland; \\ ${ }^{8}$ Department Translational Research and New Technologies, University of Pisa, Pisa, Italy: \\ ${ }^{9}$ Oslo University Hospital and Univeristy of Oslo, Oslo, Norway.
}

\begin{abstract}
Summary
Urinary Tract Infections (UTIs) are amongst the most common infectious diseases and carry a significant impact on patient quality of life and health care costs. Despite that, there is no well-established recommendation for a "standard" prophylactic antibiotic management to prevent UTI recurrences. The majority of patients undergoes long-term antibiotic treatment that severely impairs the normal microbiota and increases the risk of development of multidrugresistant microorganisms. In this scenario, the use of phytotherapy to both alleviate symptoms related to UTI and decrease the rate of symptomatic recurrences is an attractive alternative. Several recently published papers report conflicting findings and cannot give confident recommendations for the everyday clinical practice. A new approach to the management of patients with recurrent UTI might be to use nutraceuticals or phytotherapy after an accurate assessment of the patient's risk factors. No single compound or mixture has been identified so far as the best preventive approach in patients with recurrent UTI. We reviewed our non-antibiotic approach to the management of recurrent UTI patients in order to clarify the evidence-base for the commonly used substances, understand their pharmacokinetics and pharmacodynamics in order to tailor the best way to improve patient's quality of life and reduce the rate of antibiotic resistance.

Lack of a gold-standard recommendation and the risk of increasing antibiotic resistance is the reason why we need alternatives to antibiotics in the management of urinary tract infections (UTIs). A tailored approach according to bacterial characteristics and the patient risk factors profile is a promising option.
\end{abstract}

KEY WORDS: Nutraceuticals; Phytotherapy; Urinary tract infections.

Submitted 13 January 2017; Accepted 28 January 2017

\section{INTRODUCTION}

Urinary tract infections (UTIs) are amongst the most common community-acquired infectious diseases, with annual costs estimated to be higher than $\$ 1.5$ billion in the United States (1-2). The impact on public health is significant due to the high recurrence rate and the effect on patients' quality of life (4). The mortality rate must also be taken into account (reported to be as high as 1\% in men and 3\% in women due to development of pyelonephritis and urosepsis). In particular, after an initial UTI, approximately $20-30 \%$ of women with a UTI will have a second UTI within 6 months, and 3\% will experience a third UTI during that time period (4-5). In consequence, women with recurrent UTIs (rUTIs) reported a high indirect cost as a result of the number of working days lost (6). The majority of these patients are women with recurrent bacterial cystitis or acute pyelonephritis, and the causative pathogens can usually be eradicated with a short course of oral antimicrobial therapy. However, the antibiotic treatment can lead to long term impairment of the normal microbiota of the vagina and gastrointestinal tract and to the development of multidrug-resistant micro-organisms (7-9). Moreover, there is no well-established recommendation for a 'standard' prophylactic antibiotic management to prevent UTI recurrence (10). Furthermore, many women have a number of signs and symptoms of UTIs without any evidence of bacterial presence (11). In this sense, the use of phytotherapy to alleviate symptoms related to UTI and decrease the rate of symptomatic recurrence seems a good alternative which is nowadays more commonly used. There are many good reasons for this approach e.g. the low side-effects, low costs and a high level of compliance. Also important are the low rate of efficacy of 
standard preventive measures with subsequent patient disappointment and drop-outs $(3,12)$. Traditionally, nutraceuticals and phytotherapy have been used to prevent rUTIs in otherwise healthy women. Although the results from a number of clinical studies have supported their benefits, the efficacy of nutraceuticals and phytotherapy on prevention of rUTIs remains controversial, due to heterogeneity of published studies in terms of methodology and the reported findings such as trial design; inclusion/exclusion criteria; patient characteristics; outcome measures, as well as heterogeneity of the compounds used.

The latest Cochrane reviews on UTI management concluded as follows:

- Chinese herbal medicine alone or in conjunction with antibiotics may be beneficial for treating recurrent UTIs during the acute phase of infection and may reduce the recurrent UTI incidence for at least six months post-treatment (13).

- No significant benefit was demonstrated for probiotics compared with placebo or no treatment, but a benefit cannot be ruled out as the data were few, and derived from small studies with poor methodological reporting (3).

- Given the large number of dropouts/withdrawals from studies (mainly attributed to the acceptability of consuming cranberry products particularly juice, over long periods), and poor evidence for prevention of UTI, cranberry juice cannot currently be recommended for the prevention of UTIs (14).

The lack of a standard approach for the management of patient with recurrent UTI and the need to reduce the use of antibiotics suggest to re-think current practice in UTI management. In this perspective, the nutraceutical or phytotherapic approach to recurrent UTIs should be based on a full evaluation of the clinical situation, patient risk factors and the characteristics of the bacteria (e.g. Escherichia coli, adherent to vaginal and bladder epithelial cells). This includes assessment of symptomatic or asymptomatic bacteriuria; the disease symptoms/characteristics themselves (number or recurrences); the patient's behavior (use of a spermicide, diaphragm, delayed postcoital micturition, hormonal status; other risk factors); such as $\mathrm{ABO}$-blood-group non-secretor phenotype, bowel function and water intake $(4,15-17)$. A comprehensive assessment may guide and suggest the best nutraceutical or phytotherapic approach and improve the treatment outcome. The aim of the present manuscript is to review the published data about the role of nutraceuticals and phytotherapy in the management of patients affected by recurrent UTI and also to address compounds that can modify the patient's related risk factors and improve the treatment outcome.

\section{STATE OF THE ART:}

\section{INTERNATIONAL GUIDELINES RECOMMENDATIONS}

The latest version of European Association of Urology (EAU) guidelines on urological infections states that there are many non-antimicrobial measures for prevention of recurrent UTIs but only a few of them are based on well designed studies which are needed to make evidence-based recommendations (18). In particular, EAU guidelines highlight that there is no convincing benefit for lactobacillus products or cranberry extracts as prophylaxis of recurrent UTI (18). The Scottish Intercollegiate Guidelines Network confirmed the recommendations from the EAU guidelines, highlighting that cranberry products (juice, tablets, capsules) are not standardized and the concentrations of active ingredients are not known; hence the concentration may also fluctuate between batches of the same product (19). There is no evidence to support the effectiveness of cranberry products for treating symptomatic episodes of UTI (19). The Society of Obstetricians and Gynaecologists of Canada's guidelines suggest to inform the patients about the efficacy of cranberry products in reducing recurrent urinary tract infections (IA) (20). Moreover, they suggest to not use probiotics and vaccines due to lack of demonstrated efficacy (II-2C) (20).

\section{Take home message}

- International guidelines agree that there is no convincing benefit for the use of nutraceuticals and/or phytotherapy as prophylaxis of recurrent UTIs.

- New RCTs are needed.

\section{Actual scenario:}

\section{ALTERNATIVE NON-ANTIBIOTIC MEASURES}

Contemporary reviews identify four alternative compounds for non-antibiotic management of recurrent UTIs:

- Cranberry

- Probiotics

- Chinese herbal medicine

- D-mannose

\section{Cranberry extracts}

A Cochrane review from 2008 concluded that cranberry products significantly reduced the incidence of UTIs at 12 months (RR 0.65, 95\% CI 0.46-0.90) compared with placebo/control in women with recurrent UTIs (21). In a more recent Cochrane review it was concluded that cranberry products did not significantly reduce the occurrence of symptomatic UTI in women with recurrent UTIs (14). The changed view is based on the inclusion of two additional trials in their meta-analysis (14). In these two RCTs the effectiveness of cranberry extract was compared with low-dose antibiotic prophylaxis (14). McMurdo et al. concluded that trimethoprim had a limited advantage over cranberry extract in the prevention of recurrent UTIs in older women (22). In addition Beerepoot et al. showed that cranberry capsules are less effective than low-dose $(480 \mathrm{mg}$ ) trimethoprim/sulfamethoxazole in the prevention of recurrent UTIs in premenopausal women (23). Vaccinium macrocarpon, named also American cranberry, is a rich source of polyphenols that show several in vitro properties, including antibacterial, antiviral, antimutagenic, anticarcinogenic, antitumorigenic, antiangiogenic, anti-inflammatory, and antioxidant effects (27). Moreover, cranberry extract is a promising therapy due to the fact that it is 
an anti-adhesive agent against E. coli strains and thus prevent the development of antibiotic resistance (2). On the other hand, it has been demonstrated that uropathogenic bacteria may have an intestinal origin and the intestinal tract could be an alternative site where the active components of cranberry extract may interact with E. coli decreasing its infectivity (24). Several authors demonstrated that some extracts from cranberry decrease the pathogenicity of Proteus mirabilis by limiting urothelial cell invasion and improving the mucosal immunity to uropathogens (25-26).

\section{Probiotics}

Probiotics are defined as "a preparation of, or a product containing viable, defined micro-organisms in sufficient numbers, which alter the microflora (by implantation or colonization) in a host compartment and by that exert beneficial health effects in this host" $(3,28)$. The hypothesis is that probiotics are able to establish a barrier against infectious pathogens ascending the urinary tract, colonizing, and subsequently causing infection (3, 29). The latest Cochrane review showed that probiotics were not superior to placebo in reducing the risk of recurrent symptomatic bacterial UTI. The conclusion was based on 6 studies with (352 participants (RR 0.82, 95\% CI 0.60 to $1.12 ; \mathrm{I} 2=23 \%$ ). Hence the data were derived from small studies with poor methodological quality (3). On the other hand the reported evidences could not rule out a reduction or increase in recurrent UTI in women with recurrent UTI who use prophylactic probiotics. One RCT did not provide sufficient evidence to conclude on the effect of probiotics versus antibiotics (3).

\section{Chinese herbal medicine}

Chinese herbal medicine is a part of Traditional Chinese Medicine and represents a complex of herbal formulae usually comprising 10 to 15 different herbs (13). Chinese herbal medicine formulae may be standardized or individualized according to specific needs and patients' characteristics (13). The hypothesis supporting the efficacy of Chinese herbal medicine is based on in vitro research suggesting that some commonly used Chinese herbs may confer significant diuretic, antibiotic, immune enhancing, antipyretic, anti-inflammatory and pain relieving effects (13). The latest Cochrane review, including 7 RCTs with a total of 542 women, concluded that Chinese herbal medicine alone or in conjunction with antibiotics may be useful in treating recurrent UTIs during the acute phase of an infection and may reduce the incidence of recurrent UTI for at least six months post-treatment (13). However, this evidence is based on a small number of poor quality studies with severe bias that should be taken into account.

\section{D-mannose}

D-mannose is a sugar that has an important role in human metabolism, especially in the glycosylation of certain proteins. The hypothesis is that D-mannose inhibits bacterial adherence to uroepithelial cells (30). In fact, in vitro and in vivo animal studies demonstrated that D-mannose can inhibit the adhesion of Type 1 fimbriae of the uropathogenic bacteria to uroepithelial cells (31). Only one RCT evaluated its effect as preventive agent in recurrent UTI. Kranjecec et al., in a study of 98 women, showed that patients in D-mannose group and Nitrofurantoin group had a significantly lower risk of recurrent UTI episode during prophylactic therapy compared to patients in the no prophylaxis group (RR 0.239 and $0.335, \mathrm{P}<0.0001$ ) (30). These preliminary findings are promising, but supplementary clinical trials are essential.

\section{Take home messages}

- Cranberry products did not significantly reduce the occurrence of symptomatic UTI in women with recurrent UTIs.

- Probiotics are not superior to placebo in reducing the risk of recurrent symptomatic bacterial UTI.

- Chinese herbal medicine may be useful for treating recurrent UTIs during the acute phase of infection and may reduce the incidence of recurrent UTI for at least six months post-treatment. This evidence is based on a small number of patients and low quality studies.

- No well-done studies have been performed about the use of D-mannose in preventing recurrence among UTI patients.

\section{TOO MANY ANTIBIOTICS: DOCTORS NEED TO CHANGE COURSE!}

Increasing antimicrobial resistance has stimulated interest in non-antibiotic prophylaxis of recurrent urinary tract infections. This is particularly due to the recent alarming increase of the extended spectrum beta-lactamase (ESBL)-producing Enterobacteriaceae prevalence among outpatients with UTI (32-33). Thus, antimicrobial resistance has become a major worldwide health problem during recent years. Several strategies have been developed to reduce the alarming increase of resistant bacteria (34-36). Phytotherapy and nutraceuticals may be considered an alternative approach for reducing the recurrences among UTI patients. In fact, patients with recurrent UTIs are increasingly asking their healthcare professionals about the value of taking non-antibiotic products. The non-antibiotic approach, however, should be based on a correct and deep knowledge about the real efficacy and tolerability of phytotherapy compounds. All physicians should update themselves on the latest evidences on non-antibiotic approaches to recurrent UTIs and suggest products according to international guidelines and latest reviews. The main arguments for using a non-antibiotic approach to prevent recurrence among UTI patients are as follows: The use of antibiotic prophylaxis for recurrent UTI is not generally accepted; the risk of bacterial resistance is already high and still increasing; the tolerability and the compliance to nonantibiotic products is high; preliminary study findings are promising. While long-term antibiotic prophylaxis might efficiently inhibit new intravesical bacterial growth during the treatment period, infections are likely to reccur when the prophylactic treatment ends (37-38).

Take home messages

- Contemporary antimicrobial resistance rate is alarming. 
- Phytotherapy and nutraceuticals may be considered a feasible strategy to reduce indiscriminate use of antibiotics.

\section{ASSESSMENT OF PATIENTS' BEHAVIOUR AND BACTERIAL RELATED RISK FACTORS}

Hooton et al. developed a simple risk prediction model based on the number of days with intercourse per week and the use of contraceptives (diaphragm and spermicide) for predicting the risk of UTI recurrence (39). Hooton showed that an unmarried, 24-year-old female university student who had sexual intercourse had a risk of UTI that was 2.6-fold greater than that of a similar student who had no intercourse in the previous week (39). Hooton was a pioneer in highlighting the role of riskpredicting tools in the management of women with recurrent UTIl. Recently, Cai et al. developed and validated a nomogram that accurately predicts the recurrence risk of urinary tract infection at 12 months, and which can assist in identifying women at high risk of symptomatic recurrence that can be suitable candidates for a prophylactic strategy (40). In this model they found the following independent predictors of UTI recurrence: number of sexual partners, bowel function, type of pathogens isolated (Gram-positive/negative), hormonal status, number of UTI recurrences and previous treatment of asymptomatic bacteriuria (40). In this model they excluded use of food supplements because of the high variability of products used. Cai et al. also found that the most important factors affecting the risk of new recurrences were: bowel function, presence of Gram negative strains, asymptomatic bacteriuria treatment and having had 3 or more than 3 UTI episodes (40). The importance of bowel function for development of UTI is well known and is related to the fecal-perineal-urethral contamination by enteric bacteria (40). The risk is of UTI is increased by constipation as demonstrated by LoeningBaucke et al. (41). The same authors demonstrated that constipation treatment results in the disappearance of recurrent urinary tract infections in all patients who had no anatomical abnormality of the urinary tract (41). What about Gram negatives? Cai et al. found that the presence of Gram-negative bacteria (E. coli) is associated with a higher likelihood of recurrence (40) due to three reasons:

- E. coli is the most common pathogen in UTI patients (1).

- E. coli isolated from recurrent UTI has the greatest propensity of the uropathogenic bacteria to adhere to the uroepithelial cells of women with recurrent UTI as compared with bacteria from women without recurrent infection (42).

- E. coli is able to adhere to the bladder epithelium, invade the cells and form intracellular bacterial communities, which can remain quiescent reservoirs able to cause new recurrences when reactivated (38).

Recently, Cai et al. found that antibiotic treatment of asymptomatic bacteriuria in young women with recurrent UTI is not only unnecessary, but harmful (8-9). This is due to the fact that in women undergoing antibiotic treatment, the rate of E. coli decreased over time, whereas the prevalence of E. faecalis increased gradually, sug- gesting that $E$. faecalis might be an important defense mechanism that effectively interferes with the establishment of many important enteric pathogens, such as $E$. coli (8-9). In this sense, the use of non-antibiotic approach should be preferred because it doesn't interfere with the normal commensal bowel flora.

\section{RISK FACTOR ASSESSMENT AS A GUIDE TO TREATMENT}

Assessment of the risk for recurrence is not only important for predicting the probability of a new recurrence but also for the development of a tailored therapeutic approach on the basis of patient's characteristics. A constipated patient, for example, should be informed about the relationship between the constipation and the risk of new UTI. A nutraceutical or phytotherapic approach acting on the bowel function should be preferred. Moreover, a patient who reported more than 3 episodes of previous UTI and correlation with sexual intercourses should be treated with nutraceutical products that are able to interfere with the E. coli adhesion to urothelial cell and invasion.

Furthermore, a correct non-antibiotic approach should work against one or more of the following infection mechanisms:

- Reduction of the bacterial load in the intestinal reservoir with concomitant correction of the constipation

- Reduction of the spread of E. coli from the perineal zone to the urethra and bladder.

- Reduction of the adhesion and invasion of uropathogens to the urothelial cells.

- Mobilization of the immune system against the intracellular bacterial communities avoiding reactivation and new symptomatic recurrences.

We have no an ideal nutraceutical or phytotherapic compound so far that is able to act in all the above ways to decrease the risk of recurrence. Even if cranberry extracts seem to be able to act along more than one pathway, there are limitations due to the pharmacokinetic and pharmacological characteristics of this compound. As discussed above, Cranberry extracts are able to reduce the adherence of E. coli to the urothelial cells and reduce the adherence of bacterial strains also to the intestinal cells. However, possible reasons for the inefficacy of cranberry extracts found in the cited RCTs should also be taken into account. Firstly, multi-step processing of cranberry production leads to a substantial loss of phytochemicals (through elimination of rich fractions like skin and seeds), which are further damaged by thermal degradation, as well as oxidation by polyphenol oxidase and peroxidase (38). Moreover, the exact concentration of active metabolites on the action site and the pharmacokinetics of cranberry extracts is unknown. There are other plant extracts that have shown interesting results in the prevention of UTI recurrences. Rafsanjany et al. demonstrated that a mixture of extracts from Betula spp. (birch), Orthosiphon stamineus (Java tea) and Urtica spp. (nettles) is able to reduce the adherence of the pathogen to the host cell. The same results have been obtained by Cai et al. who demonstrated that a compound with solidago, orthosiphon, birch and cranberry extracts is able 
to reduce the microbial colonization in patients with indwelling urinary catheters (43). However, this mixture is not able to cover all mechanisms of infection.

\section{Conclusions}

The lack of a gold-standard for the treatment of recurrent UTIs and the risk of provoking resistances among the pathogenic bacteria are two important reasons why we need non-antibiotic alternatives for the management of recurrent UTIs. A promising alternative is a tailored approach based on a comprehensive assessment of patient related risk factor and the characteristics of the causative pathogen. Better knowledge about the action of natural substances will enable us to develop targeted non-antibiotic prevention of recurrent urinary tract infections.

\section{AUTHOR CONTRIBUTIONS}

Cai $T$. designed the study; Tamanini I. performed the research; Cai $T$. and Tamanini I. wrote the paper; Wagenlehner F., Kulchavenya E., Tandogdu Z., Bonkat G., Bartoletti R. and Bjerklund Johansen T. revised the paper. Language Revision: Prof. John Denton from Department of Modern Philology, University of Florence, revised the paper.

\section{References}

1. Flores-Mireles AL, Walker JN, Caparon M, Hultgren SJ. Urinary tract infections: epidemiology, mechanisms of infection and treatment options. Nat Rev Microbiol. 2015; 13:269-84.

2. Tao Y, Pinzón-Arango PA, Howell AB, Camesano TA. Oral consumption of cranberry juice cocktail inhibits molecular-scale adhesion of clinical uropathogenic Escherichia coli. J Med Food. 2011; 14:739-45

3. Schwenger EM, Tejani AM, Loewen PS. Probiotics for preventing urinary tract infections in adults and children Cochrane Database Syst Rev. 2015; 12:CD008772.

4. Silverman JA, Schreiber HL 4th, Hooton TM, Hultgren SJ. From physiology to pharmacy: developments in the pathogenesis and treatment of recurrent urinary tract infections. Curr Urol Rep. 2013; 14:448-56.

5. Foxman B. Epidemiology of urinary tract infections: incidence, morbidity, and economic costs. Dis Mon. 2003; 49:53-70.

6. François M, Hanslik T, Dervaux B, et al. The economic burden of urinary tract infections in women visiting general practices in France: a cross-sectional survey. BMC Health Serv Res. 2016; 16:365.

7. Kostakioti M, Hultgren SJ, Hadjifrangiskou M. Molecular blueprint of uropathogenic Escherichia Coli virulence provides clues toward the development of anti virulence therapeutics. Virulence. 2012; 3:592-594.

8. Cai T, Mazzoli S, Mondaini N, et al. The role of asymptomatic bacteriuria in young women with recurrent urinary tract infections: to treat or not to treat? Clin Infect Dis. 2012; 55:771-7.

9. Cai T, Nesi G, Mazzoli S, et al. Asymptomatic bacteriuria treatment is associated with a higher prevalence of antibiotic resistant strains in women with urinary tract infections. Clin Infect Dis. 2015; 61:1655-61.
10. Nosseir SB, Lind LR, Winkler HA. Recurrent uncomplicated urinary tract infections in women: a review. J Womens Health. 2012; 12:347-354.

11. Chuang FC, Kuo HC. Increased urothelial cell apoptosis and chronic inflammation are associated with recurrent urinary tract infection in women PLoS One. 2013; 8:e63760.

12. Frumenzio E, Maglia D, Salvini E, et al. Role of phytotherapy associated with antibiotic prophylaxis in female patients with recurrent urinary tract infections. Arch Ital Urol Androl. 2013; 85:197-9.

13. Flower A, Wang LQ, Lewith $G$, et al. Chinese herbal medicine for treating recurrent urinary tract infections in women. Cochrane Database Syst Rev. 2015; 6:CD010446

14. Jepson RG, Williams G, Craig JC. Cranberries for preventing urinary tract infections. Cochrane Database Syst Rev. 2012; 10:CD001321

15. Adatto K, Doebele KG, Galland L, Granowetter L. Behavioral factors and urinary tract infection. JAMA. 1979; 241:2525-6.

16. Czaja CA, Stamm WE, Stapleton AE, et al. Prospective cohort study of microbial and inflammatory events immediately preceding escherichia coli recurrent urinary tract infection in women. J Infect Dis. 2009; 200:528-36.

17. Wagenlehner FM, Vahlensieck W, Bauer HW, et al. Prevention of recurrent urinary tract infections. Minerva Urol. Nefrol. 2013; 65:9-20.

18. European Association of Urology Guidelines - http://uroweb.org/ wp-content/uploads/19-Urological-infections_LR2.pdf - access on August 2016.

19. http://www.sign.ac.uk/guidelines/fulltext/88/section3.html\#3_5_1 - Scottish Intercollegiate Guidelines Network - Healthcare Improvement Scotland, Gyle Square, 1 South Gyle Crescent, Edinburgh EH12 9EB. access on August 2016.

20. http://sogc.org/wp-content/uploads/2013/01/gui250CPG1011E _001.pdf - Society of Obstetricians and Gynaecologists of Canada guidelines - access on August 2016.

21. Jepson RG, Craig JC. Cranberries for preventing urinary tract infections. Cochrane Database Syst Rev. 2008; 1:CD001321.

22. McMurdo ME, Argo I, Phillips G, et al. Cranberry or trimethoprim for the prevention of recurrent urinary tract infections? A randomized controlled trial in older women. J. Antimicrob. Chemother. 2009; 63:389-395.

23. Beerepoot MA ter RG, Nys $S$, van derWal WM, et al. Cranberries vs. antibiotics to prevent urinary tract infections: A randomized double-blind noninferiority trial in premenopausal women. Arch. Intern. Med. 2011; 171:1270-1278.

24. Johnson JR, Russo TA, Brown JJ, Stapleton A. PapG alleles of Escherichia coli strains causing first-episode or recurrent acute cystitis in adult women. J Infect Dis. 1998; 12:97-101.

25. Hopkins WJ, Elkahwaji J, Beierle LM, et al. Vaginal mucosal vaccine for recurrent urinary tract infections in women: results of a phase 2 clinical trial. J Urol. 2007; 12:1349-1353.

26. Amalaradjou Mar, Narayanan A, Venkitanarayanan K. Transcinnamaldehyde decreases attachment and invasion of uropathogenic Escherichia coli in urinary tract epithelial cells by modulating virulence gene expression. J Urol. 2011; 12:1526-1531.

27. Blumberg JB, Camesano TA, Cassidy A, et al. Cranberries and their bioactive constituents in human health. Adv Nutr. 2013; 4:618-32. 
28. Schrezenmeir J, de Vrese M. Probiotics, prebiotics, and synbiotics - approaching a definition. Am J Clin Nutr. 2001; 73(2 Suppl):361S-364S

29. Bruce AW, Reid G. Intravaginal instillation of lactobacilli for the prevention of recurrent urinary tract infections. Can Journal Microbiol. 1988; 34:339-43.

30. Kranjčec B, Papeš D, Altarac S. D-mannose powder for prophylaxis of recurrent urinary tract infections in women: a randomized clinical trial. World J Urol. 2014; 32:79-84.

31. Altarac S, Papes D. Use of D-mannose in prophylaxis of recurrent urinary tract infections (UTIs) in women. BJU Int. 2014; 113:9-10

32. Al-Mayahie S, Al Kuriashy JJ. Distribution of ESBLs among Escherichia coli isolates from outpatients with recurrent UTIs and their antimicrobial resistance. J Infect Dev Ctries 2016; 10:575-83.

33. Gupta K, Scholes D, Stamm WE. Increasing prevalence of antimicrobial resistance among uropathogens causing acute uncomplicated cystitis in women. JAMA. 1999; 281:736-738. [

34. Cai T, Verze P, Brugnolli A, et al. Adherence to European Association of Urology Guidelines on Prophylactic Antibiotics: An Important Step in Antimicrobial Stewardship. Eur Urol. 2016; 69:276-83

35. Charani E, Cooke J, Holmes A. Antibiotic stewardship programmes--what's missing? J Antimicrob Chemother. 2010; 65:2275-7.
36. Cai T, Mazzoli S, Mondaini N, et al. The role of asymptomatic bacteriuria in young women with recurrent urinary tract infections: to treat or not to treat? Clin Infect Dis. 2012; 55:771-7.

37. Eells SJ, Bharadwa K, McKinnell JA, Miller LG. Recurrent urinary tract infections among women: Comparative effectiveness of 5 prevention and management strategies using a Markov chain Monte Carlo model. Clin Infect Dis. 2014; 58:147-160.

38. Lüthje P, Brauner A. Novel Strategies in the Prevention and Treatment of Urinary Tract Infections. Pathogens. 2016; 27:5.

39. Hooton TM, Scholes D, Hughes JP, et al. A prospective study of risk factors for symptomatic urinary tract infection in young women. N Engl J Med. 1996; 335:468-74.

40. Cai T, Mazzoli S, Migno S, et al. Development and validation of a nomogram predicting recurrence risk in women with symptomatic urinary tract infection. Int J Urol. 2014; 21:929-34.

41. Loening-Baucke $V$. Urinary incontinence and urinary tract infection and their resolution with treatment of chronic constipation of childhood. Pediatrics. 1997; 100:228-32.

42. Schaeffer AJ, Jones JM, Falkowski WS, et al. Variable adherence of uropathogenic Escherichia coli to epithelial cells from women with recurrent urinary tract infection. J Urol. 1982; 128:1227-30.

43. Cai T, Caola I, Tessarolo F, et al. Solidago, Orthosiphon, Birch and Cranberry extracts can decrease microbial colonization and biofilm development in indwelling urinary catheter: a microbiologic and ultrastructural pilot study. World J Urol. 2014; 32:1007-14.

\section{Correspondence}

Tommaso Cai, MD (Corresponding Author)

ktommy@libero.it

Irene Tamanini, MD

Department of Urology, Santa Chiara Regional Hospital,

Largo Medaglie d'Oro 9 - 38123 Trento, Italy

Ekaterina Kulchavenya, MD

TB Research Institute, Novosibirsk 630040, Russia

Tamara Perepanova, MD

S.R. Urology Institute, Moscow 105425, Russia

Béla Köves, MD

Jahn Ferenc South Pest Teaching Hospital, 1204 Budapest, Hungary

Florian M.E. Wagenlehner, MD

Klinik und Poliklinik für Urologie, Kinderurologie und Andrologie,

Universitätsklinikum Giessen und Marburg GmbH, Justus-Liebig-

Universität, 35392, Giessen, Germany

Zafer Tandogdu, MD

Northern Institute for Cancer Research, Newcastle University,

NE1 7RU Newcastle upon-Tyne, UK

Gernot Bonkat, MD

Department of Urology, University Hospital Basel, University of Basel, Basel, Switzerland

Riccardo Bartoletti, MD

Dept. Translational Research and New Technologies, University of Pisa, 56121 - Pisa, Italy

Truls E. Bjerklund Johansen,MD

Department of Urology, Oslo University Hospital, 0424 Oslo, Norway 